\title{
On reverse Hilbert-type inequalities
}

\author{
Biao $\mathrm{Xu}^{1 *}$, Xu-Huan Wang ${ }^{2}$, Wei Wei ${ }^{3}$ and Haoxiang Wang ${ }^{4}$
}

"Correspondence: xubiao512@163.com

'School of Mathematical Science, Huaibei Normal University, Huaibei, 235000, P.R. China

Full list of author information is available at the end of the article

\begin{abstract}
By introducing two pairs of conjugate exponents and estimating the weight coefficients, we establish reverse versions of Hilbert-type inequalities, as described by Jin (J. Math. Anal. Appl. 340:932-942, 2008), and we prove that the constant factors are the best possible. As applications, some particular results are considered.
\end{abstract}

Keywords: reverse Hilbert-type inequality; weight coefficient; best constant factor

\section{Introduction}

If both $a_{n}$ and $b_{n} \geq 0$, such that $0<\sum_{n=1}^{\infty} a_{n}^{2}<\infty$ and $0<\sum_{n=1}^{\infty} b_{n}^{2}<\infty$, then we have (see [1])

$$
\sum_{n=1}^{\infty} \sum_{m=1}^{\infty} \frac{a_{m} b_{n}}{m+n}<\pi\left\{\sum_{n=1}^{\infty} a_{n}^{2}\right\}^{\frac{1}{2}}\left\{\sum_{n=1}^{\infty} b_{n}^{2}\right\}^{\frac{1}{2}},
$$

where the constant factor $\pi$ has the best possible value. Inequality (1.1) is the well-known Hilbert inequality, introduced in 1925; inequality (1.1) has been generalized by Hardy as follows.

If $p>1, \frac{1}{p}+\frac{1}{q}=1$, and both $a_{n}$ and $b_{n} \geq 0$, such that $0<\sum_{n=1}^{\infty} a_{n}^{p}<\infty$ and $0<\sum_{n=1}^{\infty} b_{n}^{q}<$ $\infty$, then we have

$$
\sum_{n=1}^{\infty} \sum_{m=1}^{\infty} \frac{a_{m} b_{n}}{m+n}<\frac{\pi}{\sin (\pi / p)}\left\{\sum_{n=1}^{\infty} a_{n}^{p}\right\}^{\frac{1}{p}}\left\{\sum_{n=1}^{\infty} b_{n}^{q}\right\}^{\frac{1}{q}},
$$

where the constant factor $\frac{\pi}{\sin (\pi / p)}$ is the best possible. Inequality (1.2) is the well-known Hardy-Hilbert inequality, which is important in analysis and applications (see [2]). In recent years, many results with generalizations of this type of inequality have been obtained (see [3]).

Under the same conditions as in (1.2), there are some Hilbert-type inequalities that are similar to (1.2), which also have been studied and generalized by some mathematicians.

Recently, by studying a Hilbert-type operator, Jin [4] obtained a new bilinear operator inequality with the norm, and he provided some new Hilbert-type inequalities with the best constant factor. First, we repeat the results of [4].

Definition 1.1 Let $H_{p, q}(r, s)$ be the set of functions $k(x, y)$ satisfying the following conditions.

@2014 Xu et al.; licensee Springer. This is an Open Access article distributed under the terms of the Creative Commons Attribution License (http://creativecommons.org/licenses/by/2.0), which permits unrestricted use, distribution, and reproduction in any medium, provided the original work is properly cited. 
Let $p>1, \frac{1}{p}+\frac{1}{q}=1, r>1, \frac{1}{r}+\frac{1}{s}=1$, suppose that $k(x, y)$ is continuous in $(0, \infty) \times(0, \infty)$ and satisfies:

(1) $k(x, y)=k(y, x)>0$, where $x, y \in(0, \infty)$.

(2) For $\varepsilon \geq 0$ and $x>0$, the function $k(x, t)\left(\frac{x}{t}\right)^{\frac{1+\varepsilon}{l}}(l=r, s)$ is decreasing in $t \in(0, \infty)$.

For $\varepsilon \geq 0$ small enough, $x>0$, and $\bar{k}_{l}(\varepsilon, x)$ can be written as

$$
\bar{k}_{l}(\varepsilon, x):=\int_{0}^{\infty} k(x, t)\left(\frac{x}{t}\right)^{\frac{1+\varepsilon}{l}} d t \quad(l=r, s),
$$

where $\bar{k}_{l}(\varepsilon, x)$ is independent of $x, \bar{k}_{l}(0, x):=\int_{0}^{\infty} k(x, t)\left(\frac{x}{t}\right)^{\frac{1}{l}} d t=k_{p}(l=r, s), k_{p}$ is a positive constant independent of $x$, and $\bar{k}_{l}(\varepsilon, x)=k_{p}(\varepsilon)=k_{p}+o(1)\left(\varepsilon \rightarrow 0^{+}\right)$.

$$
\text { (3) } \begin{aligned}
\sum_{m=1}^{\infty} \frac{1}{m^{1+\varepsilon}} \int_{0}^{1} k(m, t)\left(\frac{m}{t}\right)^{\frac{1+\varepsilon(s / q)}{s}} d t=O(1) \quad\left(\varepsilon \rightarrow 0^{+}\right), \\
\sum_{m=1}^{\infty} \frac{1}{m^{1+\varepsilon}} \int_{0}^{1} k(m, t)\left(\frac{m}{t}\right)^{\frac{1+\varepsilon(r / p)}{r}} d t=O(1) \quad\left(\varepsilon \rightarrow 0^{+}\right) .
\end{aligned}
$$

We have Jin's result as follows.

Theorem 1.1 If $p>1, \frac{1}{p}+\frac{1}{q}=1, r>1, \frac{1}{r}+\frac{1}{s}=1$, and $k(x, y) \in H_{p, q}(r, s), a_{n}, b_{n} \geq 0$, such that $0<\sum_{n=1}^{\infty} n^{\frac{p}{r}-1} a_{n}^{p}<\infty$ and $0<\sum_{n=1}^{\infty} n^{\frac{q}{s}-1} b_{n}^{q}<\infty$, then we have

$$
\begin{aligned}
& \sum_{n=1}^{\infty} \sum_{m=1}^{\infty} k(m, n) a_{m} b_{n}<k_{r}\left\{\sum_{n=1}^{\infty} n^{\frac{p}{r}-1} a_{n}^{p}\right\}^{\frac{1}{p}}\left\{\sum_{n=1}^{\infty} n^{\frac{q}{s}-1} b_{n}^{q}\right\}^{\frac{1}{q}}, \\
& \sum_{n=1}^{\infty} n^{\frac{p}{r}-1}\left[\sum_{m=1}^{\infty} k(m, n) a_{m}\right]^{p}<\left(k_{r}\right)^{p} \sum_{n=1}^{\infty} n^{\frac{p}{r}-1} a_{n}^{p} .
\end{aligned}
$$

Here the constant factors $k_{r}$ and $\left(k_{r}\right)^{p}$ are the best possible. Inequality (1.3) is equivalent to $(1.4)$.

If $p=r$ and $q=s$ in Theorem 1.1, then Theorem 1.1 reduces to Yang's result [5] as follows.

Theorem 1.2 If $p>1, \frac{1}{p}+\frac{1}{q}=1, k(x, y) \in H(p, q)$, and both $a_{n}$ and $b_{n} \geq 0$, such that $0<$ $\sum_{n=1}^{\infty} a_{n}^{p}<\infty$ and $0<\sum_{n=1}^{\infty} a_{n}^{p}<\infty$, then we have

$$
\begin{aligned}
& \sum_{n=1}^{\infty} \sum_{m=1}^{\infty} k(m, n) a_{n} b_{n}<k_{p}\left\{\sum_{n=1}^{\infty} a_{n}^{p}\right\}\left\{\sum_{n=1}^{\infty} b_{n}^{q}\right\}^{\frac{1}{q}}, \\
& \sum_{n=1}^{\infty}\left[\sum_{n=1}^{\infty} k(m, n) a_{m}\right]^{p}<\left(k_{p}\right)^{p} \sum_{n=1}^{\infty} a_{n}^{p},
\end{aligned}
$$

where the constant factors $k_{p}$ and $\left(k_{p}\right)^{p}$ are the best possible. Inequality (1.3) is equivalent to $(1.4)$.

In this paper, by introducing some parameters, we establish a reverse version of the inequality (1.3). As applications, some particular results are considered. 


\section{Some lemmas}

Definition 2.1 Let $H_{p, q}(r, s)$ be the set of functions $k(x, y)$ satisfying the following conditions:

Let $0<p<1, \frac{1}{p}+\frac{1}{q}=1, r>1, \frac{1}{r}+\frac{1}{s}=1$, suppose that $k(x, y)$ is continuous in $(0, \infty) \times(0, \infty)$ and satisfies:

(1) $k(x, y)=k(y, x)>0, x, y \in(0, \infty)$.

(2) For $\varepsilon \geq 0$ and $x>0$, the function $k(x, t)\left(\frac{x}{t}\right)^{\frac{1+\varepsilon}{l}}(l=r, s)$ is decreasing in $t \in(0, \infty)$.

For $\varepsilon \geq 0$ small enough, for $x>0, \bar{k}_{l}(\varepsilon, x)$ can be described as

$$
\bar{k}_{l}(\varepsilon, x):=\int_{0}^{\infty} k(x, t)\left(\frac{x}{t}\right)^{\frac{1+\varepsilon}{l}} d t \quad(l=r, s)
$$

where $\bar{k}_{l}(\varepsilon, x)$ is independent of $x$, and $\bar{k}_{l}(0, x):=\int_{0}^{\infty} k(x, t)\left(\frac{x}{t}\right)^{\frac{1}{l}} d t=k_{p}(l=r, s), k_{p}$ is a positive constant independent of $x$, and $\bar{k}_{l}(\varepsilon, x)=k_{p}(\varepsilon)=k_{p}+o(1)\left(\varepsilon \rightarrow 0^{+}\right)$.

(3) There exists a positive constant $\lambda^{\prime}$ such that

$$
\begin{aligned}
& \theta_{\lambda}(s, m)=\frac{1}{k_{r}} \int_{0}^{1} k(m, t)\left(\frac{m}{t}\right)^{\frac{1}{s}} d t=O\left(1 / m^{\lambda^{\prime}}\right) \in(0,1) \quad(m \rightarrow \infty), \\
& \theta_{\lambda}(r, n)=\frac{1}{k_{r}} \int_{0}^{1} k(t, n)\left(\frac{n}{t}\right)^{\frac{1}{r}} d t=O\left(1 / n^{\lambda^{\prime}}\right) \in(0,1) \quad(n \rightarrow \infty) .
\end{aligned}
$$

Lemma 2.2 If $0<p<1, \frac{1}{p}+\frac{1}{q}=1, r>1, \frac{1}{r}+\frac{1}{s}=1, k(x, y) \in H_{p, q}(r, s)$, and the weight coeffcients $w(r, p, m)$ and $w(s, q, n)$ are defined as

$$
\begin{aligned}
& \omega(r, p, m)=\sum_{n=1}^{\infty} k(m, n) \frac{m^{\frac{p-1}{r}}}{n^{\frac{1}{s}}}, \\
& \omega(s, q, n)=\sum_{m=1}^{\infty} k(m, n) \frac{n^{\frac{q-1}{s}}}{m^{\frac{1}{r}}}
\end{aligned}
$$

then we have

$$
\begin{aligned}
& m^{\frac{p}{r}-1} k_{r}\left(1-\theta_{\lambda}(s, m)\right)<\omega(r, p, m)<m^{\frac{p}{r}-1} k_{r}, \\
& n^{\frac{q}{s}-1} k_{r}\left(1-\theta_{\lambda}(r, n)\right)<\omega(s, q, n)<n^{\frac{q}{s}-1} k_{r} .
\end{aligned}
$$

Proof By the assumption of the lemma, because $k(x, t)\left(\frac{x}{t}\right)^{\frac{1}{s}}(t \in(0, \infty))$ is decreasing, then we find

$$
\begin{aligned}
\omega(r, p, m) & =m^{\frac{p}{r}-1} \sum_{n=1}^{\infty} k(m, n)\left(\frac{m}{n}\right)^{\frac{1}{s}} \\
& \leq m^{\frac{p}{r}-1} \int_{0}^{\infty} k(m, t)\left(\frac{m}{t}\right)^{\frac{1}{s}} d t \\
& =m^{\frac{p}{r}-1} k_{r} .
\end{aligned}
$$


However, we find

$$
\begin{aligned}
\omega(r, p, m) & =m^{\frac{p}{r}-1} \sum_{n=1}^{\infty} k(m, n)\left(\frac{m}{n}\right)^{\frac{1}{s}} \geq m^{\frac{p}{r}-1} \int_{1}^{\infty} k(m, t)\left(\frac{m}{t}\right)^{\frac{1}{s}} d t \\
& =m^{\frac{p}{r}-1} \int_{0}^{\infty} k(m, t)\left(\frac{m}{t}\right)^{\frac{1}{s}} d t-m^{\frac{p}{r}-1} \int_{0}^{1} k(m, t)\left(\frac{m}{t}\right)^{\frac{1}{s}} d t \\
& =m^{\frac{p}{r}-1} k_{r}-m^{\frac{p}{r}-1} \int_{0}^{1} k(m, t)\left(\frac{m}{t}\right)^{\frac{1}{s}} d t \\
& =m^{\frac{p}{r}-1} k_{r}\left(1-\frac{1}{k_{r}} \int_{0}^{1} k(m, t)\left(\frac{m}{t}\right)^{\frac{1}{s}} d t\right) \\
& =m^{\frac{p}{r}-1} k_{r}\left(1-\theta_{\lambda}(s, m)\right) .
\end{aligned}
$$

It is easy to show that the above inequalities take the form of a strict inequality. Hence, we have $m^{\frac{p}{r}-1} k_{r}\left(1-\theta_{\lambda}(s, m)\right)<\omega(r, p, m)<m^{\frac{p}{r}-1} k_{r}$. Similarly, we can obtain $n^{\frac{q}{s}-1} k_{r}(1-$ $\left.\theta_{\lambda}(r, n)\right)<\omega(s, q, n)<n^{\frac{q}{s}-1} k_{r}$. The lemma is proved.

Lemma 2.3 If $0<p<1, \frac{1}{p}+\frac{1}{q}=1, r>1, \frac{1}{r}+\frac{1}{s}=1$, and $k(x, y) \in H_{p, q}(r, s)$, for $\varepsilon>0$ small enough, we have

$$
\sum_{m=1}^{\infty} \sum_{n=1}^{\infty} k(m, n) m^{-\frac{1}{r}-\frac{\varepsilon}{p}} n^{-\frac{1}{s}-\frac{\varepsilon}{q}}<(k r+o(1)) \sum_{1}^{\infty} m^{-1-\varepsilon} \quad\left(\varepsilon \rightarrow 0^{+}\right) .
$$

Proof For $\varepsilon>0$, by Definition 2.1, we have

$$
\begin{aligned}
\sum_{m=1}^{\infty} & \sum_{n=1}^{\infty} k(m, n) m^{-\frac{1}{r}-\frac{\varepsilon}{p}} n^{-\frac{1}{s}-\frac{\varepsilon}{q}} \\
& =\sum_{m=1}^{\infty} m^{-1-\varepsilon} \varepsilon \sum_{n=1}^{\infty} k(m, n)\left(\frac{m}{n}\right)^{\frac{1+\varepsilon(s / q)}{s}} \\
& \leq \sum_{m=1}^{\infty} m^{-1-\varepsilon} \int_{0}^{\infty} k(m, t)\left(\frac{m}{t}\right)^{\frac{1+\varepsilon(s / q)}{s}} d t \\
& =\sum_{m=1}^{\infty} m^{-1-\varepsilon} k r\left(\frac{\varepsilon s}{q}\right)=(k r+o(1)) \sum_{1}^{\infty} m^{-1-\varepsilon}
\end{aligned}
$$

The lemma is proved.

\section{Main results}

Theorem 3.1 If $0<p<1, \frac{1}{p}+\frac{1}{q}=1, r>1, \frac{1}{r}+\frac{1}{s}=1$, and $k(x, y) \in H_{p, q}(r, s), a_{n}, b_{n} \geq 0$, such that $0<\sum_{n=1}^{\infty} n^{\frac{p}{r}-1} a_{n}^{p}<\infty$ and $0<\sum_{n=1}^{\infty} n^{\frac{q}{s}-1} b_{n}^{q}<\infty$, then we have

$$
\begin{aligned}
& \sum_{n=1}^{\infty} \sum_{m=1}^{\infty} k(m, n) a_{m} b_{n}>k_{r}\left\{\sum_{n=1}^{\infty}\left[1-\theta_{\lambda}(s, n)\right] n^{\frac{p}{r}-1} a_{n}^{p}\right\}^{\frac{1}{p}}\left\{\sum_{n=1}^{\infty} n^{\frac{q}{s}-1} b_{n}^{q}\right\}^{\frac{1}{q}}, \\
& \sum_{n=1}^{\infty} n^{\frac{p}{r}-1}\left[\sum_{m=1}^{\infty} k(m, n) a_{m}\right]^{p}>\left(k_{r}\right)^{p} \sum_{n=1}^{\infty}\left[1-\theta_{\lambda}(s, n)\right] n^{\frac{p}{r}-1} a_{n}^{p},
\end{aligned}
$$


where the constant factor $k_{r}$ and $\left(k_{r}\right)^{p}$ are the best possible. Inequality (3.1) is equivalent to (3.2).

Proof By Hölder's inequality, we have (see [6])

$$
\begin{aligned}
\sum_{n=1}^{\infty} \sum_{m=1}^{\infty} k(m, n) a_{m} b_{n} & =\sum_{n=1}^{\infty} \sum_{m=1}^{\infty}\left\{[k(m, n)]^{\frac{1}{q}} \frac{m^{\frac{1}{q r}}}{n^{\frac{1}{p s}}} a_{m}\right\}\left\{[k(m, n)]^{\frac{1}{q}} \frac{n^{\frac{1}{p s}}}{m \frac{1}{q r}} b_{n}\right\} \\
& \geq\left\{\sum_{m=1}^{\infty} \sum_{n=1}^{\infty} k(m, n) \frac{m^{\frac{p-1}{r}}}{n^{\frac{1}{s}}} a_{m}^{p}\right\}^{\frac{1}{p}}\left\{\sum_{n=1}^{\infty} \sum_{m=1}^{\infty} k(m, n) \frac{n^{\frac{q-1}{s}}}{m \frac{1}{r}} b_{n}^{q}\right\}^{\frac{1}{q}} \\
& =\left\{\sum_{m=1}^{\infty} \omega(r, p, m) a_{m}^{p}\right\}^{\frac{1}{p}}\left\{\sum_{n=1}^{\infty} \omega(s, q, n) b_{n}^{q}\right\}^{\frac{1}{q}} .
\end{aligned}
$$

Then, by (2.3), in view of $0<p<1$ and $q<0$, we have (3.1).

For $\varepsilon>0$, setting $\bar{a}_{n}=n^{-\frac{1}{r}-\frac{\varepsilon}{q}}$ and $\bar{b}_{n}=n^{-\frac{1}{s}-\frac{\varepsilon}{q}}$, we find

$$
\begin{aligned}
& \left\{\sum_{n=1}^{\infty}\left[1-\theta_{\lambda}(s, n)\right] n^{\frac{p}{r}-1} \bar{a}_{n}^{p}\right\}^{\frac{1}{p}}\left\{\sum_{n=1}^{\infty} n^{\frac{q}{s}-1} \bar{b}_{n}^{q}\right\}^{\frac{1}{q}} \\
& =\left\{\sum_{n=1}^{\infty} n^{-1-\varepsilon}-\sum_{n=1}^{\infty} O\left(1 / n^{\lambda^{\prime}}\right) n^{-1-\varepsilon}\right\}^{\frac{1}{p}}\left\{\sum_{n=1}^{\infty} n^{-1-\varepsilon}\right\}^{\frac{1}{q}} \\
& =\sum_{n=1}^{\infty} n^{-1-\varepsilon}\left[1-\left(\sum_{n=1}^{\infty} n^{-1-\varepsilon}\right)^{-1} \sum_{n=1}^{\infty} O\left(1 / n^{\lambda^{\prime}}\right) n^{-1-\varepsilon}\right]^{\frac{1}{p}} .
\end{aligned}
$$

By virtue of (2.4), we have

$$
\begin{aligned}
& \sum_{n=1}^{\infty} \sum_{m=1}^{\infty} k(m, n) \bar{a}_{m} \bar{b}_{n} \\
& \quad=\sum_{m=1}^{\infty} \sum_{n=1}^{\infty} k(m, n) m^{-\frac{1}{r}-\frac{\varepsilon}{p}} n^{-\frac{1}{s}-\frac{\varepsilon}{q}}<(k r+o(1)) \sum_{1}^{\infty} m^{-1-\varepsilon} \quad\left(\varepsilon \rightarrow 0^{+}\right) .
\end{aligned}
$$

If the constant factor $k_{r}$ in (3.1) is not the best possible factor, then there exists a positive number $K$ (with $K>k_{r}$ ), such that (3.1) is still valid if the constant factor $k_{r}$ is replaced by $K$. In particular, by (3.4) and (3.5), we have

$$
(k r+o(1)) \sum_{1}^{\infty} n^{-1-\varepsilon}>K \sum_{n=1}^{\infty} n^{-1-\varepsilon}\left[1-\left(\sum_{n=1}^{\infty} n^{-1-\varepsilon}\right)^{-1} \sum_{n=1}^{\infty} O\left(1 / n^{\lambda^{\prime}}\right) n^{-1-\varepsilon}\right]^{\frac{1}{p}},
$$

that is,

$$
(k r+o(1))>K\left[1-\left(\sum_{n=1}^{\infty} n^{-1-\varepsilon}\right)^{-1} \sum_{n=1}^{\infty} O\left(1 / n^{\lambda^{\prime}}\right) n^{-1-\varepsilon}\right]^{\frac{1}{p}} .
$$

For $\varepsilon \rightarrow 0^{+}$, it follows that $K \leq k_{r}$, which contradicts the fact that $K>k_{r}$. Hence, the constant factor $k_{r}$ in (3.1) is the best possible. 
Setting $b_{n}$ as

$$
b_{n}:=n^{\frac{p}{r}-1}\left[\sum_{m=1}^{\infty} k(m, n) a_{m}\right]^{p-1}
$$

by (3.1), we have

$$
\begin{aligned}
\left\{\sum_{n=1}^{\infty} n^{\frac{q}{s}-1} b_{n}^{q}\right\}^{p} & =\left\{\sum_{n=1}^{\infty} n^{\frac{p}{r}-1}\left[\sum_{m=1}^{\infty} k(m, n) a_{m}\right]^{p}\right\}^{p} \\
& =\left\{\sum_{n=1}^{\infty} \sum_{m=1}^{\infty} k(m, n) a_{m} b_{n}\right\}^{p} \\
& \geq\left(k_{r}\right)^{p}\left\{\sum_{n=1}^{\infty}\left[1-\theta_{\lambda}(s, n)\right] n^{\frac{p}{r}-1} a_{n}^{p}\right\}\left\{\sum_{n=1}^{\infty} n^{\frac{q}{s}-1} b_{n}^{q}\right\}^{p-1} .
\end{aligned}
$$

Hence, we obtain

$$
\infty>\sum_{n=1}^{\infty} n^{\frac{p}{r}-1}\left[\sum_{m=1}^{\infty} k(m, n) a_{m}\right]^{p} \geq\left(k_{r}\right)^{p} \sum_{n=1}^{\infty}\left[1-\theta_{\lambda}(s, n)\right] n^{\frac{p}{r}-1} a_{n}^{p}>0 .
$$

By (3.1), both (3.6) and (3.7) take the form of a strict inequality, and we have (3.2).

However, if (3.2) is valid, by Hölder's inequality, we find

$$
\begin{aligned}
& \sum_{n=1}^{\infty} \sum_{m=1}^{\infty} k(m, n) a_{m} b_{n} \\
& \quad=\sum_{n=1}^{\infty}\left[n^{\frac{1}{q}-\frac{1}{s}} \sum_{m=1}^{\infty} k(m, n) a_{m}\right]\left[n^{\frac{1}{s}-\frac{1}{q}} b_{n}\right] \\
& \quad \geq\left\{\sum_{n=1}^{\infty} n^{\frac{p}{r}-1}\left[\sum_{m=1}^{\infty} k(m, n) a_{m}\right]^{p}\right\}^{\frac{1}{p}}\left\{\sum_{n=1}^{\infty} n^{\frac{q}{s}-1} b_{n}^{q}\right\}^{\frac{1}{q}}
\end{aligned}
$$

Then, by (3.2), we have (3.1). Hence (3.2) and (3.1) are equivalent.

If the constant factor $\left(k_{r}\right)^{p}$ in (3.2) is not the best possible, by using (3.8), we find the contradiction that the constant factor $k_{r}$ in (3.1) is not the best possible. The theorem is completed.

\section{Some particular results}

(1) Setting

$$
k(x, y)=\frac{(x y)^{\frac{\lambda-1}{2}}}{(x+y)^{\lambda}}\left(1-2 \min \left\{\frac{1}{r}, \frac{1}{s}\right\}<\lambda \leq 1+2 \min \left\{\frac{1}{r}, \frac{1}{s}\right\}\right),
$$

for $0 \leq \varepsilon<\min \left\{p\left(\frac{\lambda+1}{2}-\frac{1}{r}\right), q\left(\frac{\lambda+1}{2}-\frac{1}{s}\right)\right\}$, and for fixed $x>0$, we find (see [4])

$$
\bar{k}_{s}(\varepsilon, x) \rightarrow B\left(\frac{s(\lambda+1)-2}{2 s}, \frac{r(\lambda+1)-2}{2 r}\right)=k_{r} \quad\left(\varepsilon \rightarrow 0^{+}\right)
$$


and $\bar{k}_{s}(\varepsilon, x) \rightarrow k_{r}\left(\varepsilon \rightarrow 0^{+}\right)$

$$
\begin{aligned}
0 & <\int_{0}^{1} k(m, t)\left(\frac{m}{t}\right)^{\frac{1}{s}} d t=\int_{0}^{1} \frac{(m t)^{\frac{\lambda-1}{2}}}{(m+t)^{\lambda}}\left(\frac{m}{t}\right)^{\frac{1}{s}} d t \\
& \leq \int_{0}^{1} \frac{(m t)^{\frac{\lambda-1}{2}}}{m^{\lambda}}\left(\frac{m}{t}\right)^{\frac{1}{s}} d t=\frac{1}{\left(\frac{\lambda-1}{2}+\frac{1}{r}\right)} \frac{1}{m^{\frac{1+\lambda}{2}-\frac{1}{s}}} .
\end{aligned}
$$

Hence, $\theta_{\lambda}(s, m)=O\left(m^{\frac{1}{s}-\frac{1+\lambda}{2}}\right)$. Similarly, we obtain $\theta_{\lambda}(r, n)=O\left(n^{\frac{1}{r}-\frac{1+\lambda}{2}}\right)$. For $\varepsilon \geq 0,1-$ $2 \min \left\{\frac{1}{r}, \frac{1}{s}\right\}<\lambda \leq 1+2 \min \left\{\frac{1}{r}, \frac{1}{s}\right\}$, and fixed $x>0$, the function

$$
k(x, t)\left(\frac{x}{t}\right)^{\frac{1+\varepsilon}{l}}=\frac{(x t)^{\frac{\lambda-1}{2}}}{(x+t)^{\lambda}}\left(\frac{x}{t}\right)^{\frac{1+\varepsilon}{l}}=\frac{x^{\frac{1+\varepsilon}{l}+\frac{\lambda-1}{2}}}{(x+t)^{\lambda}} t^{\frac{\lambda-1}{2}-\frac{1+\varepsilon}{l}} \quad(l=r, s)
$$

is decreasing in $(0, \infty)$. Hence, $k(x, y) \in H_{p}(r, s)$. By Theorem 3.1, we have the following.

Corollary 4.1 If $0<p<1,1 / p+1 / q=1,1 / r+1 / s=1,1-2 \min \left\{\frac{1}{r}, \frac{1}{s}\right\}<\lambda \leq 1+2 \min \left\{\frac{1}{r}, \frac{1}{s}\right\}$, and both $a_{n}, b_{n} \geq 0$ such that $0<\sum_{n=1}^{\infty} n^{\frac{p}{r}-1} a_{n}^{p}<\infty$ and $0<\sum_{n=1}^{\infty} n^{\frac{q}{s}-1} b_{n}^{q}<\infty$, then we have

$$
\begin{aligned}
& \sum_{n=1}^{\infty} \sum_{m=1}^{\infty} \frac{(m n)^{\frac{\lambda-1}{2}}}{(m+n)^{\lambda}} a_{m} b_{n} \\
& >B\left(\frac{s(\lambda+1)-2}{2 s}, \frac{r(\lambda+1)-2}{2 r}\right)\left\{\sum_{n=1}^{\infty}\left[1-\theta_{\lambda}(s, n)\right] n^{\frac{p}{r}-1} a_{n}^{p}\right\}^{\frac{1}{p}}\left\{\sum_{n=1}^{\infty} n^{\frac{q}{r}-1} b_{n}^{q}\right\}^{\frac{1}{q}}, \\
& \sum_{n=1}^{\infty} n^{\frac{p}{r}-1}\left[\sum_{m=1}^{\infty} \frac{(m n)^{\frac{\lambda-1}{2}}}{(m+n)^{\lambda}} a_{m}\right]^{p} \\
& \quad>\left[B\left(\frac{s(\lambda+1)-2}{2 s}, \frac{r(\lambda+1)-2}{2 r}\right)\right]^{p} \sum_{n=1}^{\infty}\left[1-\theta_{\lambda}(s, n)\right] n^{\frac{p}{r}-1} a_{n}^{p},
\end{aligned}
$$

where the constant factors

$$
B\left(\frac{s(\lambda+1)-2}{2 s}, \frac{r(\lambda+1)-2}{2 r}\right) \text { and }\left[B\left(\frac{s(\lambda+1)-2}{2 s}, \frac{r(\lambda+1)-2}{2 r}\right)\right]^{p}
$$

are the best possible. Inequality (4.1) is equivalent to (4.2).

In particular, (a) for $r=q, s=p$, and $1-2 \min \left\{\frac{1}{p}, \frac{1}{q}\right\}<\lambda \leq 1+2 \min \left\{\frac{1}{p}, \frac{1}{q}\right\}$, we have

$$
\begin{aligned}
& \sum_{n=1}^{\infty} \sum_{m=1}^{\infty} \frac{(m n)^{\frac{\lambda-1}{2}}}{(m+n)^{\lambda}} a_{m} b_{n} \\
& >B\left(\frac{p(\lambda+1)-2}{2 p}, \frac{q(\lambda+1)-2}{2 q}\right)\left\{\sum_{n=1}^{\infty}\left[1-\theta_{\lambda}(p, n)\right] n^{p-2} a_{n}^{p}\right\}^{\frac{1}{p}}\left\{\sum_{n=1}^{\infty} n^{q-2} b_{n}^{q}\right\}^{\frac{1}{q}}, \\
& \sum_{n=1}^{\infty} n^{p-2}\left[\sum_{m=1}^{\infty} \frac{(m n)^{\frac{\lambda-1}{2}}}{(m+n)^{\lambda}} a_{m}\right]^{p} \\
& >\left[B\left(\frac{p(\lambda+1)-2}{2 p}, \frac{q(\lambda+1)-2}{2 q}\right)\right]^{p} \sum_{n=1}^{\infty}\left[1-\theta_{\lambda}(p, n)\right] n^{p-2} a_{n}^{p} .
\end{aligned}
$$


(b) For $r=s=2$ and $0<\lambda \leq 2$, we have

$$
\begin{aligned}
& \sum_{n=1}^{\infty} \sum_{m=1}^{\infty} \frac{(m n)^{\frac{\lambda-1}{2}}}{(m+n)^{\lambda}} a_{m} b_{n}>B\left(\frac{\lambda}{2}, \frac{\lambda}{2}\right)\left\{\sum_{n=1}^{\infty}\left[1-\theta_{\lambda}(2, n)\right] n^{\frac{p}{2}-1} a_{n}^{p}\right\}^{\frac{1}{p}}\left\{\sum_{n=1}^{\infty} n^{\frac{q}{2}-1} b_{n}^{q}\right\}^{\frac{1}{q}}, \\
& \sum_{n=1}^{\infty} n^{\frac{p}{2}-1}\left[\sum_{m=1}^{\infty} \frac{(m n)^{\frac{\lambda-1}{2}}}{(m+n)^{\lambda}} a_{m}\right]^{p}>\left[B\left(\frac{\lambda}{2}, \frac{\lambda}{2}\right)\right]^{p} \sum_{n=1}^{\infty}\left[1-\theta_{\lambda}(2, n)\right] n^{\frac{p}{2}-1} a_{n}^{p} .
\end{aligned}
$$

(2) Let

$$
k(x, y)=\frac{(x y)^{\frac{\lambda-1}{2}}}{x^{\lambda}+y^{\lambda}}\left(1-2 \min \left\{\frac{1}{r}, \frac{1}{s}\right\}<\lambda \leq 1+2 \min \left\{\frac{1}{r}, \frac{1}{s}\right\}\right) .
$$

For $0 \leq \varepsilon<\min \left\{p\left(\frac{\lambda+1}{2}-\frac{1}{r}\right), q\left(\frac{\lambda+1}{2}-\frac{1}{s}\right)\right\}$ and $x>0$, we find (see [4])

$$
\bar{k}_{s}(\varepsilon, x) \rightarrow \frac{1}{\lambda} B\left(\frac{s(\lambda+1)-2}{2 s \lambda}, \frac{r(\lambda+1)-2}{2 r \lambda}\right)=k_{r} \quad\left(\varepsilon \rightarrow 0^{+}\right),
$$

and $\bar{k}_{s}(\varepsilon, x) \rightarrow k_{r}\left(\varepsilon \rightarrow 0^{+}\right)$

$$
\begin{aligned}
\int_{0}^{1} k(m, t)\left(\frac{m}{t}\right)^{\frac{1}{s}} d t & =\int_{0}^{1} \frac{(m t)^{\frac{\lambda-1}{2}}}{m^{\lambda}+t^{\lambda}}\left(\frac{m}{t}\right)^{\frac{1}{s}} d t \\
& \leq \int_{0}^{1} \frac{(m t)^{\frac{\lambda-1}{2}}}{m^{\lambda}}\left(\frac{m}{t}\right)^{\frac{1}{s}} d t=\frac{1}{\left(\frac{\lambda-1}{2}+\frac{1}{r}\right)} m^{\frac{1}{s}-\frac{1+\lambda}{2}}
\end{aligned}
$$

Hence, $\theta_{\lambda}(s, m)=O\left(m^{\frac{1}{s}-\frac{1+\lambda}{2}}\right)$. Similarly, we can obtain $\theta_{\lambda}(r, n)=O\left(n^{\frac{1}{r}-\frac{1+\lambda}{2}}\right)$. For $\varepsilon \geq 0,1-$ $2 \min \left\{\frac{1}{r}, \frac{1}{s}\right\}<\lambda \leq 1+2 \min \left\{\frac{1}{r}, \frac{1}{s}\right\}$, and $x>0$, the function

$$
k(x, t)\left(\frac{x}{t}\right)^{\frac{1+\varepsilon}{l}}=\frac{(x t)^{\frac{\lambda-1}{2}}}{x^{\lambda}+t^{\lambda}}\left(\frac{x}{t}\right)^{\frac{1+\varepsilon}{l}}=\frac{x^{\frac{1+\varepsilon}{l}+\frac{\lambda-1}{2}}}{x^{\lambda}+t^{\lambda}} t^{\frac{\lambda-1}{2}-\frac{1+\varepsilon}{l}} \quad(l=r, s)
$$

is decreasing in $(0, \infty)$. Hence $k(x, y) \in H_{p}(r, s)$. By Theorem 3.1, we have the following corollary.

Corollary 4.2 If $0<p<1, \frac{1}{p}+\frac{1}{q}=1, r>1, \frac{1}{r}+\frac{1}{s}=1,1-2 \min \left\{\frac{1}{r}, \frac{1}{s}\right\}<\lambda \leq 1+2 \min \left\{\frac{1}{r}, \frac{1}{s}\right\}$, and both $a_{n}, b_{n} \geq 0$ such that $0<\sum_{n=1}^{\infty} n^{\frac{p}{r}-1} a_{n}^{p}<\infty$ and $0<\sum_{n=1}^{\infty} n^{\frac{q}{s}-1} b_{n}^{q}<\infty$, then we have

$$
\begin{aligned}
& \sum_{n=1}^{\infty} \sum_{m=1}^{\infty} \frac{(m n)^{\frac{\lambda-1}{2}}}{m^{\lambda}+n^{\lambda}} a_{m} b_{n} \\
& >\frac{1}{\lambda} B\left(\frac{s(\lambda+1)-2}{2 s \lambda}, \frac{r(\lambda+1)-2}{2 r \lambda}\right)\left\{\sum_{n=1}^{\infty}\left[1-\theta_{\lambda}(s, n)\right] n^{\frac{p}{r}-1} a_{n}^{p}\right\}^{\frac{1}{p}}\left\{\sum_{n=1}^{\infty} n^{\frac{q}{r}-1} b_{n}^{q}\right\}^{\frac{1}{q}}, \\
& \sum_{n=1}^{\infty} n^{\frac{p}{r}-1}\left[\sum_{m=1}^{\infty} \frac{(m n)^{\frac{\lambda-1}{2}}}{m^{\lambda}+n^{\lambda}} a_{m}\right]^{p} \\
& >\left[\frac{1}{\lambda} B\left(\frac{s(\lambda+1)-2}{2 s \lambda}, \frac{r(\lambda+1)-2}{2 r \lambda}\right)\right]^{p} \sum_{n=1}^{\infty}\left[1-\theta_{\lambda}(s, n)\right] n^{\frac{p}{r}-1} a_{n}^{p},
\end{aligned}
$$


where the constant factors

$$
\frac{1}{\lambda} B\left(\frac{s(\lambda+1)-2}{2 s \lambda}, \frac{r(\lambda+1)-2}{2 r \lambda}\right) \text { and }\left[\frac{1}{\lambda} B\left(\frac{s(\lambda+1)-2}{2 s \lambda}, \frac{r(\lambda+1)-2}{2 r \lambda}\right)\right]^{p}
$$

are the best possible. Inequality (4.7) is equivalent to (4.8).

In particular, (a) for $r=q, s=p$, and $1-2 \min \left\{\frac{1}{p}, \frac{1}{q}\right\}<\lambda \leq 1+2 \min \left\{\frac{1}{p}\right.$, $\left.\frac{1}{q}\right\}$, we have

$$
\begin{aligned}
& \sum_{n=1}^{\infty} \sum_{m=1}^{\infty} \frac{(m n)^{\frac{\lambda-1}{2}}}{m^{\lambda}+n^{\lambda}} a_{m} b_{n} \frac{1}{\lambda} B\left(\frac{p(\lambda+1)-2}{2 p \lambda}, \frac{q(\lambda+1)-2}{2 q \lambda}\right) \\
& \times\left\{\sum_{n=1}^{\infty}\left[1-\theta_{\lambda}(p, n)\right] n^{p-2} a_{n}^{p}\right\}^{\frac{1}{p}}\left\{\sum_{n=1}^{\infty} n^{q-2} b_{n}^{q}\right\}^{\frac{1}{q}}, \\
& \sum_{n=1}^{\infty} n^{p-2}\left[\sum_{m=1}^{\infty} \frac{(m n)^{\frac{\lambda-1}{2}}}{(m+n)^{\lambda}} a_{m}\right]^{p} \\
&> {\left[\frac{1}{\lambda} B\left(\frac{p(\lambda+1)-2}{2 p \lambda}, \frac{q(\lambda+1)-2}{2 q \lambda}\right)\right]^{p} \sum_{n=1}^{\infty}\left[1-\theta_{\lambda}(p, n)\right] n^{p-2} a_{n}^{p} . }
\end{aligned}
$$

(b) For $r=s=2$ and $0<\lambda \leq 2$, we have

$$
\begin{aligned}
& \sum_{n=1}^{\infty} \sum_{m=1}^{\infty} \frac{(m n)^{\frac{\lambda-1}{2}}}{m^{\lambda}+n^{\lambda}} a_{m} b_{n}>\frac{\pi}{\lambda}\left\{\sum_{n=1}^{\infty}\left[1-\theta_{\lambda}(2, n)\right] n^{\frac{p}{2}-1} a_{n}^{p}\right\}^{\frac{1}{p}}\left\{\sum_{n=1}^{\infty} n^{\frac{q}{2}-1} b_{n}^{q}\right\}^{\frac{1}{q}}, \\
& \sum_{n=1}^{\infty} n^{\frac{p}{2}-1}\left[\sum_{m=1}^{\infty} \frac{(m n)^{\frac{\lambda-1}{2}}}{m^{\lambda}+n^{\lambda}} a_{m}\right]^{p}>\left[\frac{\pi}{\lambda}\right]^{p} \sum_{n=1}^{\infty}\left[1-\theta_{\lambda}(2, n)\right] n^{\frac{p}{2}-1} a_{n}^{p} .
\end{aligned}
$$

(3) Let

$$
k(x, y)=\frac{(x y)^{\frac{\lambda-1}{2}}}{(\max \{x, y\})^{\lambda}}\left(1-2 \min \left\{\frac{1}{r}, \frac{1}{s}\right\}<\lambda \leq 1+2 \min \left\{\frac{1}{r}, \frac{1}{s}\right\}\right),
$$

for $0 \leq \varepsilon<\min \left\{p\left(\frac{\lambda+1}{2}-\frac{1}{r}\right), q\left(\frac{\lambda+1}{2}-\frac{1}{s}\right)\right\}$ and $x>0$, then we find (see [4])

$$
\bar{k}_{s}(\varepsilon, x) \rightarrow \frac{4 r s \lambda}{[r(\lambda+1)-2][s(\lambda+1)-2]}=k_{r} \quad\left(\varepsilon \rightarrow 0^{+}\right)
$$

and $\bar{k}_{r}(\varepsilon, x) \rightarrow k_{r}\left(\varepsilon \rightarrow 0^{+}\right)$

$$
\begin{aligned}
0 & <\int_{0}^{1} k(m, t)\left(\frac{m}{t}\right)^{\frac{1}{s}} d t=\int_{0}^{1} \frac{(m t)^{\frac{\lambda-1}{2}}}{(\max \{m, t\})^{\lambda}}\left(\frac{m}{t}\right)^{\frac{1}{s}} d t \\
& =\int_{0}^{1} \frac{(m t)^{\frac{\lambda-1}{2}}}{m^{\lambda}}\left(\frac{m}{t}\right)^{\frac{1}{s}} d t=\frac{1}{\left(\frac{\lambda-1}{2}+\frac{1}{r}\right)} \frac{1}{m^{\frac{1+\lambda}{2}-\frac{1}{s}}} .
\end{aligned}
$$


Hence, $\theta_{\lambda}(s, m)=O\left(\frac{1}{m^{\frac{1+\lambda}{2}-\frac{1}{s}}}\right)$. Similarly, we can obtain $\theta_{\lambda}(r, n)=O\left(\frac{1}{n^{\frac{1+\lambda}{2}-\frac{1}{r}}}\right)$. For $\varepsilon \geq 0,1-$ $2 \min \left\{\frac{1}{r}, \frac{1}{s}\right\}<\lambda \leq 1+2 \min \left\{\frac{1}{r}, \frac{1}{s}\right\}$, and $x>0$, the function

$$
k(x, t)\left(\frac{x}{t}\right)^{\frac{1+\varepsilon}{l}}=\frac{(m t)^{\frac{\lambda-1}{2}}}{(\max \{m, t\})^{\lambda}}\left(\frac{x}{t}\right)^{\frac{1+\varepsilon}{l}}=\frac{x^{\frac{1+\varepsilon}{l}+\frac{\lambda-1}{2}}}{(\max \{m, t\})^{\lambda}} t^{\frac{\lambda-1}{2}-\frac{1+\varepsilon}{l}} \quad(l=r, s)
$$

is decreasing in $(0, \infty)$. Hence, $k(x, y) \in H_{p, q}(r, s)$. By Theorem 3.1, we have the following corollary.

Corollary 4.3 If $0<p<1, \frac{1}{p}+\frac{1}{q}=1, r>1, \frac{1}{r}+\frac{1}{s}=1,1-2 \min \left\{\frac{1}{r}, \frac{1}{s}\right\}<\lambda \leq 1+2 \min \left\{\frac{1}{r}, \frac{1}{s}\right\}$, and both $a_{n}, b_{n} \geq 0$, such that $0<\sum_{n=1}^{\infty} n^{\frac{p}{r}-1} a_{n}^{p}<\infty$ and $0<\sum_{n=1}^{\infty} n^{\frac{q}{s}-1} b_{n}^{q}<\infty$, then we have

$$
\begin{aligned}
& \sum_{n=1}^{\infty} \sum_{m=1}^{\infty} \frac{(m n)^{\frac{\lambda-1}{2}}}{(\max \{m, n\})^{\lambda}} a_{m} b_{n} \\
& >\frac{4 r s \lambda}{[r(\lambda+1)-2][s(\lambda+1)-2]}\left\{\sum_{n=1}^{\infty}\left[1-\theta_{\lambda}(s, n)\right] n^{\frac{p}{r}-1} a_{n}^{p}\right\}^{\frac{1}{p}}\left\{\sum_{n=1}^{\infty} n^{\frac{q}{s}-1} b_{n}^{q}\right\}^{\frac{1}{q}}, \\
& \sum_{n=1}^{\infty} n^{\frac{p}{r}-1}\left[\frac{(m n)^{\frac{\lambda-1}{2}}}{(\max \{m, n\})^{\lambda}} a_{m}\right]^{p} \\
& >\left(\frac{4 r s \lambda}{[r(\lambda+1)-2][s(\lambda+1)-2]}\right)^{p} \sum_{n=1}^{\infty}\left[1-\theta_{\lambda}(s, n)\right] n^{\frac{p}{r}-1} a_{n}^{p} .
\end{aligned}
$$

Here the constant factors $\frac{4 r s \lambda}{[r(\lambda+1)-2][s(\lambda+1)-2]}$ and $\left(\frac{4 r s \lambda}{[r(\lambda+1)-2][s(\lambda+1)-2]}\right)^{p}$ are the best possible. Inequality (4.13) is equivalent to (4.14).

In particular, (a) for $r=q, s=p$, and $1-2 \min \left\{\frac{1}{p}, \frac{1}{q}\right\}<\lambda \leq 1+2 \min \left\{\frac{1}{p}, \frac{1}{q}\right\}$, we have

$$
\begin{aligned}
& \sum_{n=1}^{\infty} \sum_{m=1}^{\infty} \frac{(m n)^{\frac{\lambda-1}{2}}}{(\max \{m, n\})^{\lambda}} a_{m} b_{n} \\
& >\frac{4 p q \lambda}{[p(\lambda+1)-2][q(\lambda+1)-2]}\left\{\sum_{n=1}^{\infty}\left[1-\theta_{\lambda}(p, n)\right] n^{p-2} a_{n}^{p}\right\}^{\frac{1}{p}}\left\{\sum_{n=1}^{\infty} n^{q-2} b_{n}^{q}\right\}^{\frac{1}{q}}, \\
& \sum_{n=1}^{\infty} n^{p-2}\left[\sum_{m=1}^{\infty} \frac{(m n)^{\frac{\lambda-1}{2}}}{(\max \{m, n\})^{\lambda}} a_{m}\right]^{p} \\
& >\left(\frac{4 p q \lambda}{[p(\lambda+1)-2][q(\lambda+1)-2]}\right)^{p} \sum_{n=1}^{\infty}\left[1-\theta_{\lambda}(p, n)\right] n^{p-2} a_{n}^{p} .
\end{aligned}
$$

(b) For $r=s=2$ and $0<\lambda \leq 2$, we have

$$
\begin{aligned}
& \sum_{n=1}^{\infty} \sum_{m=1}^{\infty} \frac{(m n)^{\frac{\lambda-1}{2}}}{(\max \{m, n\})^{\lambda}} a_{m} b_{n}>\frac{4}{\lambda}\left\{\sum_{n=1}^{\infty}\left[1-\theta_{\lambda}(2, n)\right] n^{\frac{p}{2}-1} a_{n}^{p}\right\}^{\frac{1}{p}}\left\{\sum_{n=1}^{\infty} n^{\frac{q}{2}-1} b_{n}^{q}\right\}^{\frac{1}{q}}, \\
& \sum_{n=1}^{\infty} n^{\frac{p}{2}-1}\left[\sum_{m=1}^{\infty} \frac{(m n)^{\frac{\lambda-1}{2}}}{(\max \{m, n\})^{\lambda}} a_{m}\right]^{p}>\left(\frac{4}{\lambda}\right)^{p} \sum_{n=1}^{\infty}\left[1-\theta_{\lambda}(2, n)\right] n^{\frac{p}{2}-1} a_{n}^{p} .
\end{aligned}
$$




\section{Competing interests}

The authors declare that there is no conflict of interests regarding the publication of this paper.

\section{Authors' contributions}

All authors contributed equally and significantly in writing this article. All authors read and approved the final manuscript.

\section{Author details}

${ }^{1}$ School of Mathematical Science, Huaibei Normal University, Huaibei, 235000, P.R. China. ${ }^{2}$ Department of Education

Science, Pingxiang University, Pingxiang, 337055, P.R. China. ${ }^{3}$ School of Computer Science and Engineering, Xi'an University of Technology, Xi'an, 710048, P.R. China. ${ }^{4}$ Department of Electrical and Computer Engineering, Cornell University, 300 Day Hall, 10 East Avenue, Ithaca, NY 14853, USA.

\section{Acknowledgements}

The work is supported by Scientific Research Program Funded by Shaanxi Provincial Education Department (No. 2013JK1139), China Postdoctoral Science Foundation (No. 2013M542370), NNSFC (No. 11326161), key projects of Science and Technology Research of the Henan Education Department (No. 14A110011). The authors deeply appreciate the support.

Received: 8 November 2013 Accepted: 6 May 2014 Published: 20 May 2014

\section{References}

1. Hardy, GH, Littlewood, JE, Polya, G: Inequalities. Cambridge University Press, Cambridge (1952)

2. Mitrinovic, DS, Pecaric, JE, Fink, AM: Inequalities Involving Functions and Their Integrals and Derivatives. Kluwer Academic, Boston (1991)

3. Yang, BC, Rassias, TM: On the way of weight coefficient and research for the Hilbert-type inequalities. Math. Inequal. Appl. 6(4), 625-658 (2003)

4. Jin, JJ: On Hilbert's type inequalities. J. Math. Anal. Appl. 340, 932-942 (2008)

5. Yang, BC: On the norm of a Hilbert's type linear operator and applications. J. Math. Anal. Appl. 325, 529-541 (2007)

6. Kuang, JC: Applied Inequalities. Shandong Science and Technology Press, Jinan (2004)

\section{Submit your manuscript to a SpringerOpen ${ }^{\circ}$ journal and benefit from:}

- Convenient online submission

Rigorous peer review

- Immediate publication on acceptance

- Open access: articles freely available online

- High visibility within the field

- Retaining the copyright to your article 